\title{
Bio-intensive management of fungal diseases of fruits and vegetables utilizing compost and compost teas
}

\begin{abstract}
Compost has been used in agriculture and horticulture for a long time as a fertilizer or a soil conditioner. However, in recent years the focus has been placed on the disease-suppressive effects of the compost and its aqueous extracts. Several composts and their water extracts have been investigated on different diseases especially on those caused by soilborne pathogens throughout the world. Conversely, an increase in disease incidence has also been reported. Therefore, it remains the fact that compost application can suppress the diseases in positive, neutral, and even negative manner. This makes the utilization of compost intricate as a robust prevalent approach to mitigate plant diseases. The mechanisms involved in the disease suppression are not fully known. The disease-suppressive effect of the compost could be attributed to various mechanisms including the presence of antagonistic microbes and unidentified chemical factors in them, antibiosis, and induction of systemic resistance in plants and improvement of the overall health of plants. This chapter focuses on the influence of compost and compost teas on the suppression of foliar and soilborne fungal diseases of fruits and vegetables. The factors limiting and enhancing their efficacy will also be discussed.
\end{abstract}

Keyword: Compost; Fertilizer; Plant diseases 